\title{
Functional Standardization of Life Cycle of Information System
}

\author{
A.V. Boichenko \\ Plekhanov Russian University of Economics \\ Moscow, Russia \\ Boichenko46@mail.ru
}

\author{
O.V.Lukinova \\ Institute of Control Sciences of Russian Academy of \\ Sciences \\ Moscow, Russia \\ Lobars@mail.ru
}

\begin{abstract}
Features of three basic standards ISO/IEC 12207, ISO/IEC 15288 and GOST 34.601-90 regarding methodological approaches to management of life cycle of the designed information system are shown. Limitation of specification of the standardized kinds of activity of life cycle at the level of methods and procedures in these documents is noted. For a solution it is offered to use instruments of functional standardization in the form of technological profiles.
\end{abstract}

Keywords - management of life cycle; information system; profile of life cycle; functional standardization, profile.

\section{INTRODUCTION}

Standardization of life cycle of information systems and the software entering into them - one of key aspects of creation, operation and modernization of information systems. The concept of life cycle of software and his standardization is also a cornerstone of program engineering.

The existing standards considering life cycle of information system (or her software) entirely contain three levels of specification of stages or processes of life cycle. At the lower level semantics of stages (processes) is washed away owing to an essential difference in their semantic filling.

As the management of life cycle assumes contents rationing including the lower levels, the solution of this problem seems in use of methodology of functional standardization in the form of creation of profiles of standards of life cycle for the concrete, designed information system.

\section{METHODOLOGICAL FEATURES OF REGULATORY BASE OF MANAGEMENT OF LIFE CYCLE OF IS}

Today the regulatory base regulating the life cycle (LC) of the software (S), and also the information systems (IS) in general includes both the Russian, and foreign sources. For domestic developers of the automated systems ("information" in new terminology) for many years the state standard specification 34.601-90 [1] complex was used. And now the given standard is applied in the practical purposes.

The international organization for standardization and the International electrotechnical commission has issued a series of standards and methodological reports on program and system engineering. These are, first of all, the basic standards ISO/IEC
15288:2002, Systems engineering. System life cycle processes and ISO/IEC 12207, Information technology - Software life cycle processes, 1995. Later the following versions of the specified standards expanding subject domain of the previous versions have been released (especially it concerns ISO/IEC 12207). In due time both versions of the specified standards have been accepted by Federal Agency for Technical Regulation and Metrology of the Russian Federation as national state standard specifications, today in system of standardization of the Russian Federation are the operating state standard specifications P ISO/IEC 12207-2010 [2] and state standard specification P ISO/IEC 15288:2005 [3] (see tab. $1)$.

Table 1.

The basic standards regulating management of LC IS and software

\begin{tabular}{|l|l|}
\hline \multicolumn{1}{|c|}{ International Standard } & \multicolumn{1}{|c|}{ Russian analog } \\
\hline $\begin{array}{l}\text { ISO/IEC 12207:2008 Information } \\
\text { technology - Software life cycle } \\
\text { processes, }\end{array}$ & $\begin{array}{l}\text { GOST R } \\
\text { ISO/IEC12207-2010 } \\
\text { "Information } \\
\text { technology. System and } \\
\text { program engineering. } \\
\text { Processes of life cycle of } \\
\text { software. [Operating]. }\end{array}$ \\
\hline $\begin{array}{l}\text { ISO/IEC 15288:2002, Systems } \\
\text { engineering. System life cycle } \\
\text { processes }\end{array}$ & $\begin{array}{l}\text { GOST R ISO/IEC12207-2010 } \\
\text { "Information } \\
\text { technology. System and } \\
\text { program engineering. } \\
\text { Processes of life cycle of } \\
\text { systems. [Operating]. }\end{array}$ \\
\hline $\begin{array}{l}\text { ISO/IEC 15288:2008 Systems } \\
\text { and software engineering - Life } \\
\text { cycle processes }\end{array}$ & \begin{tabular}{l} 
There is no analog \\
\hline
\end{tabular}
\end{tabular}

From the point of view of methodological approaches to management of LC of systems, the specified documents have basic distinctions. So, GOST 34.601-90 rather rigidly establishes stages and stages of creation of information system. As units of activities for creation of IS in the standard works 
which are ordered on time and logical following by means of stages and stages are defined and reflect the LC cascade model.

Feature of this model is that transition from the previous stage to the subsequent is carried out only after full completion of works of the previous stage. Respectively at each stage the finished set of the project documentation sufficient is formed in order that development could be continued by other group of developers. Thus, synthesis of GOST 34.601.90 and cascade model attracts both the advantage, and a lack of such approach to design. The positive moment is the possibility of planning of date of completion of works and costs of their performance that allows to regulate accurately the relations between the customer and the developer. The essential shortcoming consists in lack of flexibility of cascade model, i.e. it is that it is very difficult to lay real process of creation of information system or the software in the tough scheme and therefore constantly there is a need of return to the previous stages for the purpose of specification and revision of earlier made decisions.

GOST R ISO/IEC12207 defines processes, subprocesses and tasks which regulate life cycle of software and by which it is possible to be guided during creation of software products.

The state standard GOST R ISO/IEC 15288 belongs to the field of system engineering and describes processes of LC of system of any appointment. From the point of view of information system processes of this standard can be used in case standards of direct appointment don't cover all tasks connected with management of LC IS. His harmonization with ISO/IEC 15288 therefore it contains much wider nomenclature of processes which in many respects is crossed with processes of ISO/IEC 15288 was the purpose of the publication of the ISO/IEC 12207:2008 version.

The international standards ISO/IEC 12207, ISO/IEC 15288 and the corresponding Russian analogs which were conceptually more developed are also standards of new generation. In them other idea of management of design of IS is put essentially. Unlike the tough cascade scheme ZhTs regulated by the GOST 34th state standard specification, the specified standards are interpreted as library of processes which, generally speaking, cover all space of the tasks arising during creation and maintenance of IS. But here what specifically it is necessary to solve problems when developing this or that IS i.e. what processes to choose from "library" in what sequence to build them is a business of exclusively expert judgment, is defined, first of all, by that methodology which is accepted for this development in common by the customer and the developer and the LC model. In [5] the assumption becomes that, the methodologies of creation of applied software used today is agile-methods, SCRUM methodology, DevOps-approach, microservices, extreme programming and so forth - are forms of realization of stages LC of the software.

For this reason the organizations for standardization publish the additional reports and state standard specifications [6-9] representing methodological material on application of the ISO/IEC 12207 and ISO/IEC 15288 standards and their domestic analogs.

\section{HIERARCHICAL PRINCIPLE OF CREATION OF KINDS OF ACTIVITY}

Kinds of activity on management of LC in the specified standards are constructed by the hierarchical principle. Each of them is focused on the 3rd-level specification:

1. At the top level - a stage (in accordance with GOST 34.601-90) or process according to the international standards, i.e. the part of activities for creation of IS limited to certain time frames and which is coming to an end with release of the concrete product (models, program components, documentation) determined by the requirements set for this stage / process;

2. The following level - stage/subprocess, i.e. some specification of stage/process at the substantial level;

3. The last level of specification - concrete work / task which sets represent a stage/subprocess essence.

So, the ISO/IEC 12207:2010 standard groups different types of activity which can be carried out during life cycle of program systems, in seven groups of processes: processes of the agreement, organizational support of the project, project, technical processes, processes of realization of software, supports of software, repeated application of software. Processes of GOST ISO/IEC 15288:2005 are subdivided into four groups: agreements, enterprises, project, technical processes. Each of processes of life cycle within these groups is described in terms of the purpose and desirable exits, lists of subprocesses and tasks which need to be carried out for achievement of these results; it can be begun at any moment of LC and be carried out along with any other processes, at the same time there are no regulations much their use.

GOST 34.601-90 breaks all LC into eight stages: formation of requirements to the automation system, development of the concept the automation system, the specification, the outline sketch, the engineering design, working documentation, commissioning, maintenance the automation systemwhich include the corresponding stages and works. At the same time it is allowed to exclude a stage "Outline sketch", separate stages of works at all stages, to unite stages "Engineering design" and "Working documentation" in one stage "the working project".

The problem is that if for the first level of activity standards contain rather substantial interpretation (though there are exceptions), then for deyatelnost of the lower levels semantics is often washed away. And it is clear, the essence of the performed works is too variable here, depends on many factors: technologies of development, specifics of the developed IS, options of configuration of life cycle, etc.

The decision seems in use of methodology of the functional standardization (FS) which assumes breakdown of difficult object on parts and the description of separate parts the harmonized standards or specifications. Such description is called a profile. According to [10] profile is the set of several (or a subset of one) basic standards (and other normative documents) with accurately certain and harmonized subsets of obligatory and facultative opportunities intended for realization of the set function or group of functions. Strictly speaking, in 
[10] the object of profiling is understood as information system for which description the functional profile is formed. However it is obvious that LC IS not less difficult object, than IS and, certainly, it can be object of profiling, and instructions to his structurization at the standard level offer the ZhTs standards considered above. For such object of profiling in [11,12] the concept of a technological or auxiliary profile is entered. Thus, set of the standards and specifications defining the set of processes (stages) of LC adapted for concrete IS, maintenance and application of these processes defines a profile of life cycle or the IS technological profile.

This set of standards has to be ordered on the basis of the chosen LC model as which can be used cascade model, cascade model with intermediate control (evolutionary, iterative) or spiral model.

\section{CREATION OF A TECHNOLOGICAL PROFILE}

The algorithm of creation of a technological profile of life cycle of IS (profile of life cycle of IS) consists of the following steps:

1. First of all it is necessary to choose the basic standard within which modeling of LC and its management will be carried out. Options here following: a). if modeling of LC is made only under the developer, then the profile should be built from the very beginning under regulatory base of the ISO/IEC 12207 standard; б). if development of IS custom, then is more expedient to use both GOST 34.601-90, and ISO/IEC 12207.

2. Further it is necessary to choose the ZhTs model. If the cascade model, then for GOST 34.601-90 it "native" is chosen, processes of ISO/IEC 12207 and ISO/IEC 15288:2005 it is necessary to have so that they kept within linear structure. For comparison, the iterative and spiral LC models more flexible therefore they are suitable for process methodology more.

3. The following step it is necessary to develop the chosen model in time, i.e. to construct Gant's chart structured according to the LC model. It will allow to exercise control of the project both from the customer, and from the developer, and also to record points of communication with the customer and to define timepoints when it is necessary to prepare a set of reporting documents on the project.

4. The most important step - arrangement on Gant's chart of those stages / processes, stages/subprocesses, works/tasks of life cycle, the list and which configuration, actually, also reflect specifics of this project and regarding the used methodology of programming and regarding functional features of IS. It will provide base for management of ZhTs of concrete IS, and also are a standard basis for creation of a technological profile of life cycle of the developed system.

5. Further it is possible to start creation of a profile. For this purpose for each kind of activity reflected in Gant's chart we select the corresponding standard. If the kind of activity is rather difficult and isn't described by one standard, it is necessary to use several suitable, but at the same time they have to be harmonized, i.e. not contain contradictions, and are limited if necessary. If standards in the field don't exist, it is necessary to develop own specification and to include it in a profile. For example, process of documenting can be standardized only regarding the user documentation, but how to standardize the description formation of requirements regulatory base isn't regulated.

6. At last the profile should be issued in the form of the document. It can be done in two ways:

a). in the form of the standardized documentation which scheme is provided in [10], the being main normative document of functional standardization;

b) in any format (for example, in the form of table), the only requirement is in that the hierarchy of kinds of activity has been completely reflected and everyone them them surely has to be standardized one way or another.

Thus, the profile as the main instrument of functional standardization, serves for unification, a regulation, adaptation and a specification of set of basic standards in relation to certain classes of projects, functions, processes and tasks of LC IS. Purpose of a technological profile consists in the following:

1. Fixings of concrete classes, subsets, facultative opportunities and parameters of basic standards for ensuring controllability of the project of development of IS;

2. Planning of volumes of the resources necessary for implementation of the project both from developers, and from the customer;

\section{Ensuring control of performance of work of the project;}

4. Creation of the typified reference standard materials for various professional categories: developers, suppliers of program and technical means, users.

\section{CONCLUSION}

In article the comparative analysis of the main standards allowing exercising control of LC IS is carried out. Methodological distinctions in approaches to modeling of LC are considered, it is noted that at their practical use there are three main problems: a). inflexibility of GOST 34.601-90 that doesn't allow to conduct development in a paradigm of a version control of the final product; b). absence of the formalized criteria of the choice of kinds of activity when modeling LC; c). absence in standards of completeness and a reality in the description of kinds of activity, especially the 3rd level. It is shown that the third problem can be "closed" if to add the kinds of activity specified on Gant's chart of the LC model with sets of the harmonized and limited standards and/or specifications which list make the LC IS profile.

\section{REFERENCES}

1. GOST 34.601-90. The automated systems. Creation stages.

2. GOST R ISO/IEC 12207-2010. Information technology. Processes of life cycle of software.

3. GOST P ISO IEC 15288-2005. Information technology. System engineering. Processes of life cycle of systems.

4. Boichenko A.V., Lukinova O. V. Application of the POSIX OSE/RM model at creation of subsystems of 
information security//Works of the International scientific and practical conferences "Intellectual Systems" (AIS'10) and "Intellectual SAPR" (CAD-2010). - 2010. - T. 2. - Page 473476.

5. Boichenko A.V. New calls for design of information systems//Works VII of the international scientific and practical conference "Electronic Kazan". - 2015.

6. ISO/IEC TR 19760:2003 Systems engineering -- A guide for the application of ISO/IEC 15288 (System life cycle processes) по применению ISO/IEC 15288.

7. ISO/IEC TR 15271, Information technology - Guide for ISO/IEC 12207. 1998.

8. GOST P ISO/IEC TR 15271-2002. Information technology. Guide to ISO/IEC 12207.

9. GOST P ISO/IEC TR 16326-2002. Program engineering. An application guide of state standard specification P ISO/IEC 12207 at management of the project.

GOST P ISO IEC TR 10000-1-99 Information technology. Bases and taxonomy of the international functional standards. Part 1. General provisions and bases of documenting. 1999.

11. Boychenko A.V., Filinov E.N. Problems and technique of formation of profiles of open information systems//Director of information service. - 2001. - No. 8.

12. Lipayev V. V., Filinov E.N. Formation and application of profiles of open information systems//Open systems. - 1997. - No. 5. 
